Research report

\title{
Increased neural resources recruitment in the intrinsic organization in major depression
}

\author{
Yuan Zhou ${ }^{\mathrm{a}, \mathrm{b}}$, Chunshui Yuc,*, Hua Zheng ${ }^{\mathrm{d}}$, Yong Liu ${ }^{\mathrm{b}}$, Ming Song ${ }^{\mathrm{b}}$, Wen Qin ${ }^{\mathrm{c}}$, \\ Kuncheng $\mathrm{Li}^{\mathrm{c}}$, Tianzi Jiang ${ }^{\mathrm{b}, *}$ \\ a Center for Social and Economic Behavior, Institute of Psychology, Chinese Academy of Sciences, Beijing, PR China \\ b National Laboratory of Pattern Recognition, Institute of Automation, Chinese Academy of Sciences, Beijing, PR China \\ ' Department of Radiology, Xuanwu Hospital of Capital Medical University, Beijing, PR China \\ d Department of Neurology, Xuanwu Hospital of Capital Medical University, Beijing, PR China
}

\section{A R T I C L E I N F O}

\section{Article history:}

Received 16 November 2008

Received in revised form 26 May 2009

Accepted 26 May 2009

Available online 21 June 2009

\section{Keywords:}

Depression

Functional connectivity

Functional magnetic resonance imaging

Resting state

\begin{abstract}
A B S T R A C T
Objective: To investigate the functional connectivity (FC) pattern within an intrinsic functional organization, including both task-positive (TPN) and task-negative (TNN) networks, in major depressive disorder (MDD), and to examine relationships between the involved FCs and clinical variables.

Methods: Resting-state FC analyses were used to identify the component brain regions of the intrinsic organization and to investigate the FCs of the individual component regions in 18 firstepisode, medication-naïve MDD and 20 healthy control subjects.

Results: We found that the intrinsic organization of the depressed group recruited more extensive regions than the control group. All of the altered FCs associated with the component regions increased in MDD. Specifically, in the TPN the increased FCs were primarily located in the bilateral lateral prefrontal cortices and the inferior parietal lobes, which have been implicated in attention and adaptive control. In the TNN, the increased FCs were primarily located in the posterior cingulate cortex and the medial orbitofrontal cortex, which are involved in episodic memory, self-reflection and emotional regulation. We also found increased anticorrelations between the two networks. Additionally, the strengths of the FCs associated with the lateral prefrontal cortices were found to be correlated with the duration of the depressive episode and the HDRS scores in the depressed patients.

Limitations: Clinical correlates of these abnormal FCs should be cautiously interpreted due to the small sample size in this study.

Conclusions: Abnormalities in the intrinsic organization may be an underlying basis for the pronounced and prolonged negative bias in processing emotional information observed in MDD.
\end{abstract}

(C) 2009 Elsevier B.V. All rights reserved.

\section{Introduction}

Major depressive disorder (MDD) is characterized by persistent and overwhelming feelings of sadness, guilt, and

\footnotetext{
* Corresponding authors. Jiang is to be contacted at National Laboratory of Pattern Recognition, Institute of Automation, Chinese Academy of Sciences, Beijing 100190, PR China. Tel.: +8610 8261 4469; fax: +86 10 62551993. Yu, Department of Radiology, Xuanwu Hospital of Capital Medical University, Beijing 100053, PR China. Tel./fax: +86 1083198376.

E-mail addresses: chunshuiyu@yahoo.cn (C. Yu), jiangtz@nlpr.ia.ac.cn (T. Jiang).
}

worthlessness (American Psychiatric Association, 2000). The production of depression may depend on a distributed neuronal network consisting of cortical and limbic regions rather than on the activity of a discrete brain region (Davidson et al., 2002; Drevets, 2000; Drevets et al., 2008; Mayberg, 2003; Phillips et al., 2003; Seminowicz et al., 2004). Therefore investigating the functional connectivity (FC) pattern of the brain is helpful for increasing our understanding of the pathophysiology of MDD.

The tonic nature of these core depressive symptoms indicates that resting-state imaging studies may be valuable. 
Several recent studies have found decreased FCs in the cortico-limbic circuit (Anand et al., 2005a,b) and increased FCs within the default mode network (Greicius et al., 2007) in MDD during rest. Although the default mode network is a part of an intrinsic functional organization of the brain, which has recently been proposed (Fox et al., 2005; Fransson, 2005), no studies of MDD have systematically investigated resting-state FCs within the intrinsic functional organization of brain.

Functional magnetic resonance (fMRI) studies have found that during rest under healthy conditions the brain operates within a certain functional organization to process ongoing spontaneous activities (Fox and Raichle, 2007). The intrinsic functional organization, composed of a task-positive network (TPN) and a task-negative network (TNN) (Fox et al., 2005), is thought to play a role in spontaneously modulating the ongoing information processing activities during rest, such as monitoring the external and internal environment which includes the emotional state (Fransson, 2005; Gusnard and Raichle, 2001),. Specifically, the TPN includes a set of regions, such as the dorsolateral prefrontal cortex (DLPFC), the inferior parietal lobule (IPL), the middle temporal region $(\mathrm{MT}+)$ and the supplementary motor cortex (SMA) (Corbetta and Shulman, 2002), which routinely show increased activation during attention demanding cognitive tasks. These regions also show coherence in spontaneous activity during rest (Fox et al., 2006) and have together been considered to constitute the network known as the TPN. The TNN includes another set of regions, such as the posterior cingulate cortex (PCC) and retrosplenial cortex, the medial prefrontal cortex (MPFC), the hippocampus/parahippocampal gyrus and the lateral parietal cortex (LPC), which are routinely activated during tasks related to self-reflective activity (Frith and Frith, 2006), emotional processing (Maddock et al., 2003) and episodic memory retrieval (Andreasen et al., 1995). Coherent activity of these regions has repeatedly been observed during rest (Greicius et al., 2003; Gusnard et al., 2001; Raichle et al., 2001). Because these regions routinely show decreased activity during cognitive tasks (Shulman et al., 1997), they are termed the TNN or the default mode network. The TPN and TNN show a temporally anti-correlated relationship (Fox et al., 2005; Fransson, 2005). Reports of high degrees of spontaneous, temporal anti-correlation between these networks strongly indicate a potentially high degree of antagonism between the psychological functions which the TPN and TNN reflect (for a review, see Sonuga-Barke and Castellanos, 2007). Several studies have suggested that the anti-correlation between the two networks may prove to be functionally more important than the TNN/default mode network activity itself (Fox et al., 2005; Uddin et al., 2009; Broyd et al., 2009). Altogether, the intrinsic organization of correlations within each network and the anti-correlations between the two networks may play an important role in the coordination of and competition between ongoing information processing activities.

The FCs in the intrinsic functional organization have been found to be altered in psychiatric disorders other than MDD. A recent study of schizophrenia found that the increased interregional FCs in the intrinsic organization are widely distributed within the TPN and TNN and between the two networks (Zhou et al., 2007b), but an autism study only found disrupted FCs within the TN (Kennedy and Courchesne,
2008). Increasing empirical research into altered activity patterns in the intrinsic functional organization in psychiatric disorders has increased the confidence of researchers in the potential clinical relevance of the TNN/default mode network and its anti-correlated network in building contemporary models of psychopathology (for a review, please see Broyd et al., 2009). Investigating the correlations within each network and the anti-correlations between networks in the intrinsic functional organization underlying information processing may help to reveal the neural basis of psychiatric disorders.

Since the intrinsic organization includes many brain regions involved in emotion processing, attention and episodic memory, and since a negative bias in the processing of emotional information (including attentional bias and memory bias) is a pronounced and prolonged characteristic of MDD (Leppanen, 2006), we hypothesize that the intrinsic organization may be altered in MDD. Specifically, (1) the persistent, emotion-laden, self-reflective tendencies and the enhanced memory of negative emotional material in depressed patients may be reflected by altered functional connectivities (FCs) within the TNN; (2) the tendency to have an attention bias toward negative emotional material may be caused by altered FCs within the TPN; and (3) the anticorrelation between the two networks may change with the altered correlation within each network. In order to test this hypothesis, we performed a resting-state FC analysis, which measured the correlations of spontaneous low frequency fluctuations (SLFF) BOLD signal between a seed region and all brain voxels. This method has been widely used in restingstate fMRI studies in other psychiatric disorders (Allen et al., 2007; Bluhm et al., 2007; Wang et al., 2006; Zhou et al., 2007a), and can provide detailed information on specific changes in FCs in such disorders. In this study, we first used the resting-state FC analysis to identify the component brain regions of the intrinsic organization and then to investigate differences in the FCs of these component regions between medication-naïve patients with a first depressive episode and healthy control subjects.

\section{Methods and materials}

\subsection{Subjects}

Patients with a first major depressive episode were recruited from the outpatient clinic at Xuanwu Hospital. Demographically matched healthy subjects were recruited via advertisement. All subjects met the following criteria: age 1860 years, no history of substance abuse or dependence, no family history of major psychiatric or neurological illness in first degree relatives, not currently taking any prescription or centrally acting medications, no serious medical or neurological illness, and satisfying the criteria for undergoing an MRI scan based on an MRI screening questionnaire. The patients satisfied the Diagnostic and Statistical Manual fourth edition (DSM-IV) criteria for a major depressive episode, single episode, currently depressed, according to the Structured Clinical Interview for DSM-IV (First et al., 1995) and had never taken any psychotropic medication. A follow-up observation further confirmed the diagnosis. The patients were excluded if they had any preexisting or concurrent co-morbid primary 
diagnosis that met the DSM-IV criteria for any Axis I disorder other than MDD. Additional exclusion criteria were acutely suicidal or homicidal or requiring inpatient treatment, history of trauma resulting in loss of consciousness, and current pregnancy or breastfeeding. Similar exclusion criteria were adopted for healthy subjects. Immediately before scanning, the depressive symptoms of the patients were rated using the 17-item Hamilton Depression Rating Scale (HDRS) (Hamilton, 1960). The end result was that we recruited twenty depressed patients and 20 healthy subjects. Two patients were later excluded due to excessive head motion during the fMRI scan (see the following section). The remaining patients did not significantly differ from the healthy subjects in age and gender distribution (Table 1). All subjects took part in this study after signing an informed consent form approved by the Medical Research Ethics Committee of Xuanwu Hospital.

\subsection{Image acquisition}

MR images sensitized to changes in BOLD signal levels were obtained by an echo planar imaging sequence on a 3.0Tesla Siemens MR scanner (repetition time $=2000 \mathrm{~ms}$; echo time $=30 \mathrm{~ms}$; flip angle $=90^{\circ}$, matrix $=64 \times 64$; field of view $=220 \times 220 \mathrm{~mm}^{2}$; slice thickness $=3 \mathrm{~mm}$; slice gap $=$ $1 \mathrm{~mm}$, and thus acquisition voxel size $=3.4 \times 3.4 \times 4 \mathrm{~mm}^{3}$ ). Each brain volume was comprised of 32 axial sections and each functional run contained 180 volumes. During the fMRI data acquisition, all subjects were instructed to keep their eyes closed, relax and move as little as possible. Highresolution T1-weighted images were acquired in a saggital orientation employing a magnetization prepared rapid gradient echo (MP-RAGE) sequence (repetition/echo time = 2000/2.6 ms; flip angle $=9$ degrees; slice thickness $=1 \mathrm{~mm}$, no gap) with a voxel size of $1 \times 1 \times 1 \mathrm{~mm}^{3}$.

\subsection{Data preprocessing}

Unless specifically stated otherwise, all the preprocessing steps were carried out using statistical parametric mapping (SPM2, http://www.fil.ion.ucl.ac.uk/spm). After the first 10 volumes of each functional time series were discarded to allow for magnetization equilibrium, the remaining 170 volumes were conventionally preprocessed using the following steps: slice timing, motion correction, spatial normalization to the standard MNI space and resampling to $3 \times 3 \times 3 \mathrm{~mm}^{3}$, followed by spatial smoothing with $4 \mathrm{~mm}$ full-width at half maximum Gaussian kernel.

Table 1

Demographic and clinical details.

\begin{tabular}{llll}
\hline & $\begin{array}{l}\text { Normal } \\
\text { controls }\end{array}$ & $\begin{array}{l}\text { Depressed } \\
\text { patients }\end{array}$ & $p$ \\
\hline Number of subjects & 20 & 18 & \\
Gender (male/female) & $6 / 14$ & $4 / 14$ & $0.43^{\mathrm{a}}$ \\
Age (years) & $40.6 \pm 10.7$ & $38.9 \pm 9.9$ & $0.63^{\mathrm{b}}$ \\
Duration of current episode (months) & & $7.0 \pm 4.1$ & \\
HDRS & & $16.7 \pm 3.7$ & \\
Mean displacement (mm) & $0.22 \pm 0.13$ & $0.21 \pm 0.11$ & $0.7^{\mathrm{b}}$ \\
Peak displacement (mm) & $0.48 \pm 0.28$ & $0.4 \pm 0.2$ & $0.3^{\mathrm{b}}$ \\
\hline
\end{tabular}

\footnotetext{
a Pearson Chi-square two-tailed test.
}

b Two-tailed $t$-test.
Based on their recorded motion correction estimates, two subjects who had more than $1.5 \mathrm{~mm}$ maximum displacement in any of the $x, y$ or $z$ directions or more than $1.5^{\circ}$ of angular rotation about any axis for any of the 170 volumes were excluded from this study. Because correlation analysis is sensitive to gross head motion effects, we further characterized the mean displacement and peak displacement as measures of head motion for each subject (Jiang et al., 1995; Lowe et al., 1998). The remaining 18 patients did not significantly differ from the healthy subjects in head motion measures (Table 1). To further reduce the effects of confounding factors, six motion parameters, linear drift and the mean time series of all voxels in the whole brain were removed from the data through linear regression (Fox et al., 2005). Finally, temporal band-pass filtering (0.01-0.08 Hz) was performed on the time series of each voxel using the AFNI (http://www.afni.nimh.nih.gov/) 3D Fourier program to reduce the effects of low frequency drift and high-frequency noise (Biswal et al., 1995).

\subsection{Reconstruction of the intrinsic organization}

The intrinsic organization was reconstructed as previously described (Zhou et al., 2007b). Briefly, the reconstruction of this intrinsic organization was done in four steps:

1. Extraction of the time series of the seed regions: the right DLPFC (BA46 in the right middle frontal gyrus), a region in the TPN, and the PCC/PCu (BA31 in the bilateral posterior cingulate cortices and the adjacent precuneus), a region in the TNN, were generated using the free software WFU_PickAtlas (http://www.ansir.wfubmc.edu) (Maldjian et al., 2003). The BOLD time series of the voxels within each seed region were averaged to generate the reference time series for this seed region.

2. Resting-state correlation maps of the seed regions: for each subject and each seed region (right DLPFC and PCC/ $\mathrm{PCu}$ ), a correlation map was produced by computing the correlation coefficients (Pearson's $r$ ) between the reference time series and the time series of every voxel in the brain. To combine results across subjects and compute statistical significance, correlation coefficients were converted to a normal distribution by Fischer's $r$-to- $z$ transformation (Press et al., 1992). Then, Fischer z maps were combined across subjects by using a random-effects analysis (one sample $t$-test for the patient group and the normal group, separately). This method obtained the brain regions that showed significant positive or negative correlations with the seed region within each group $(q<.05$, False Discovery Rate correction) (Genovese et al., 2002).

3. Reconstruction of the intrinsic organization: within each group, by intersecting the regions having significantly positive correlations with the right DLPFC with those having significantly negative correlations with the PCC/ PCu using the ImCalc toolbox in SPM2, we obtained the regions belonging to the TPN Similarly, by intersecting the regions that had significantly negative correlations with the right DLPFC with those that had significantly positive correlations with the $\mathrm{PCC} / \mathrm{PCu}$, we obtained the regions belonging to the TNN for each group (Zhou et al., 2007b). 
In this manner we obtained the regions that constitute the intrinsic organization for each group. We only report the clusters that contain more than 25 voxels.

4. Mask: after obtaining the regions that constitute the intrinsic organization for each group, a binary mask was created by setting the value of voxels within the intrinsic organization to 1 , and setting the value of voxels outside the intrinsic organization to 0 . Thus a control mask and a patient mask were generated separately. Then a combined mask was generated by combining the control mask and patient mask. All of these were done using the ImCalc toolbox in SPM2.

\subsection{Descriptive analysis of the intrinsic organization pattern}

By intersecting the control mask and the patient mask, the overlap between the two groups was obtained. Within both the patient group and the normal group, the overlap percentage of each region was computed as the cluster size of the overlap divided by the total cluster size of this region. Depending on its overlap percentage, each region could be classified into one of three types.

Specifically, if the overlap percentage of a region was greater than $50 \%$ in both the control group (overlap ${ }_{\mathrm{NC}}>50 \%$ ) and the depressed group (overlap $\mathrm{MD}_{\mathrm{MD}}>50 \%$ ), the contribution of the region to the constitution of the intrinsic organization in the control group was considered to be "similar" to that in the depressed group. Otherwise, this region was classified into either "NC" (normal control) or "MD" (major depression) types, depending on which group had the larger area that did not overlap. Thus the group that had the larger area that did not overlap was considered to be the predominant group for this region. Because these regions appeared in both of the groups, but with different percentages of overlap, the regions were further classified as group-common network components. If a region only appeared in one group (no overlap), the region was classified as a group-specific network component.

\subsection{FC analyses of the intrinsic organization}

A voxel-wise FC analysis was performed separately for each region that constituted the intrinsic organization. To obtain patient-specific connectivity patterns, the overlapping portion of each group-common or the group-specific network component was selected as the seed region. Correlation maps were produced by extracting the BOLD time series from each seed region and then computing the correlation coefficients

Table 2

Regions constituting the intrinsic pattern within the normal control group and within the depressed group.

\begin{tabular}{|c|c|c|c|c|c|c|c|c|}
\hline \multirow[t]{2}{*}{ Index } & \multirow[t]{2}{*}{ Region } & \multirow[t]{2}{*}{ BA } & \multicolumn{3}{|c|}{ Cluster size } & \multirow[t]{2}{*}{ Overlap $_{\mathrm{NC}}$} & \multirow[t]{2}{*}{ Overlap $_{\mathrm{MD}}$} & \multirow[t]{2}{*}{ Type } \\
\hline & & & $\mathrm{NC}$ & MD & Overlap & & & \\
\hline \multicolumn{9}{|l|}{ TPN } \\
\hline 1 & R. DLPFC & $9 / 46 / 10$ & 449 & 610 & 382 & 85.1 & 62.6 & similar \\
\hline 2 & L. DLPFC & $9 / 46 / 10$ & 460 & 637 & 362 & 78.7 & 56.8 & similar \\
\hline 3 & R. IFG & $44 / 45$ & 477 & 402 & 318 & 66.7 & 79.1 & similar \\
\hline 4 & L. IFG & $44 / 45$ & 276 & 281 & 163 & 59.1 & 58 & similar \\
\hline 5 & R. latOFG & 11 & 52 & 40 & 26 & 50 & 65 & similar \\
\hline 6 & L. latOFG & 11 & 49 & 51 & 23 & 46.9 & 45.1 & MD \\
\hline 7 & R. insula & $13 / 47$ & 410 & 158 & 106 & 25.9 & 67.1 & $\mathrm{NC}$ \\
\hline 8 & L. insula & $13 / 47$ & 263 & 87 & 70 & 26.6 & 80.5 & $\mathrm{NC}$ \\
\hline 9 & R. IPL & $40 / 2$ & 543 & 659 & 345 & 63.5 & 52.4 & similar \\
\hline 10 & L. IPL & $40 / 2$ & 560 & 838 & 363 & 64.8 & 43.3 & MD \\
\hline 11 & R. MT+ & 37 & 98 & 124 & 65 & 66.3 & 52.4 & similar \\
\hline 12 & L. MT+ & $37 / 19$ & 91 & 101 & 21 & 23.1 & 20.8 & MD \\
\hline 13 & SMA & $32 / 6$ & 103 & 150 & 50 & 48.5 & 33.3 & MD \\
\hline \multirow[t]{2}{*}{14} & R. dPM & 6 & 133 & 69 & 32 & 24.1 & 46.4 & $\mathrm{NC}$ \\
\hline & L. mid-posterior cingulate cortex & 31 & - & 28 & & - & 100 & $\mathrm{MD}^{\mathrm{a}}$ \\
\hline 15 & R. inferior cerebellum & - & 26 & 49 & 15 & 57.7 & 30.6 & MD \\
\hline 16 & L. inferior cerebellum & - & 53 & 37 & 28 & 52.8 & 75.7 & similar \\
\hline \multicolumn{9}{|l|}{ TNN } \\
\hline 17 & $\mathrm{PCC} / \mathrm{PCu}$ & $31 / 7 / 30$ & 1245 & 1197 & 958 & 77 & 80 & similar \\
\hline 18 & $\mathrm{MPFC} / \mathrm{mOFC}$ & $9 / 10 / 11 / 32$ & 536 & 962 & 443 & 82.6 & 46 & MD \\
\hline 19 & R. mSFG & 8 & 69 & 159 & 35 & 50.7 & 22 & MD \\
\hline \multirow[t]{2}{*}{20} & L. $\mathrm{mSFG}$ & 8 & 267 & 157 & 101 & 37.8 & 64.3 & $\mathrm{NC}$ \\
\hline & sgACC & $11 / 25$ & - & 153 & & - & 100 & $\mathrm{MD}^{\mathrm{a}}$ \\
\hline 21 & R. LPC & 39 & 32 & 186 & 13 & 40.6 & 7 & MD \\
\hline 22 & L. LPC & 39 & 72 & 264 & 60 & 83.3 & 22.7 & MD \\
\hline 23 & R. anterior temporal cortex & $21 / 20$ & 155 & 373 & 117 & 75.5 & 31.4 & MD \\
\hline \multirow[t]{2}{*}{24} & L. anterior temporal cortex & $21 / 20$ & 214 & 584 & 181 & 84.6 & 31 & MD \\
\hline & R. parahippocampal gyrus & - & - & 101 & & - & 100 & $\mathrm{MD}^{\mathrm{a}}$ \\
\hline 25 & L. parahippocampal gyrus & - & 31 & 145 & 5 & 16.1 & 3.4 & MD \\
\hline 26 & R. cerebellum (uvula) & - & 98 & 59 & 40 & 40.8 & 67.8 & $\mathrm{NC}$ \\
\hline 27 & L. cerebellum (uvula) & - & 27 & 64 & 19 & 70.4 & 29.7 & MD \\
\hline 28 & Cerebellum (tonsil) & - & 144 & 125 & 80 & 55.6 & 64 & similar \\
\hline
\end{tabular}

Abbreviations: BA: Brodmann area; L: left; R: right; DLPFC: dorsolateral frontal gyrus; IFG: inferior frontal gyrus; labOFC: lateral orbitofrontal gyrus; IPL: inferior parietal lobe; MT+: middle temporal region; SMA: supplementary motor area; dPM: dorsal premotor area; PCC/PCu: posterior cingulate cortex/precuneus; MPFC/mOFC: medial prefrontal cortex/orbitofrontal cortex; mSFG: medial superior frontal gyrus; sgACC: subgenual anterior cingulate cortex; LPC: lateral parietal cortex; OverlapNC: the percentage of the overlap part of a region in the control group; OverlapMD: the percentage of the overlap part of a region in the depressed group.

a The region only appeared in the depressed group not in the normal control group. 
between the reference time series and the time series from all voxels within the combined mask. This study focused on the differences in FCs of the component region within the combined mask between groups rather than on the pattern of the FCs of the component region within the combined mask in each group. Thus, after the Fisher's $r$-to- $z$ transform, the $z$ values for each individual were entered into a twosample $t$-test to determine the differences in FCs within the combined mask between the two groups. A combined threshold of contrast maps was set at $p<0.01$ for each voxel and a cluster size of at least $675 \mathrm{~mm}^{3}$, which was equal to the corrected threshold of $p<0.001$, determined by a Monte Carlo simulation (see program AlphaSim by B.D. Ward in AFNI software. Parameters were: single voxel $p=0.01$, $\mathrm{FWHM}=4 \mathrm{~mm}$, with combined mask, http://afni.nimh.nih. gov/).

\subsection{Clinical correlates analysis}

To take into consideration the effect of age on the restingstate FC (Andrews-Hanna et al., 2007), a partial correlation controlling for the age effect was used to explore the clinical correlates of the altered FCs. Partial correlation coefficients were computed between the mean $z$ values of clusters that showed altered FCs in the FC difference map of each seed region and each of the two clinical variables (HDRS score and current depressive episode duration) after partialling out age in depressed patients. For each seed region, if the number of the altered connectivity was $n$, then the number of correlation analyses was $2 \times n$. Following the Bonferroni correction for multiple comparisons, the level of significance was established at a $p$ equal or less than $0.05 / 2 \times n$.

\section{Results}

3.1. Differences in the component regions of the intrinsic organization between groups

In the control group, the TPN consisted of 16 regions, including the bilateral DLPFC, inferior frontal gyrus (IFG),
OFG, insula, IPL, MT+, inferior cerebellum, SMA and right dorsal premotor area. The TNN consisted of 12 regions, including the PCC extending to the precuneus ( $\mathrm{PCC} / \mathrm{PCu})$, the MPFC extending to the orbitofrontal cortex (MPFC/mOFC), the bilateral medial superior frontal gyrus (mSFG), the LPC, the anteriortemporal cortex, the left parahippocampus gyrus and three cerebellar regions (the tonsil and the bilateral uvula) (Table 2, Fig. 1). These results are consistent with previous studies (Fox et al., 2005; Fransson, 2005; Zhou et al., 2007b). These regions also appeared in the intrinsic organization of the depressed group (Table 2, Fig. 1). According to the percentage of overlap of each region within each group, 10 regions were classified into the "similar" type, 5 regions into the "NC" type, and 13 regions into the "MD" type. Thus there were 28 group-common network components (Table 2).

In addition, we found three group-specific network components. These three regions (the left mid-posterior cingulate cortex in the TPN, the subgenual anterior cingulate cortex (sgACC) and right parahippocmapus gyrus in the TNN) only appeared in the depressed patients based on the threshold that we used (Table 2). We did not find any region that only appeared in the healthy subjects.

\subsection{Differences in the FCs of the component regions of the intrinsic organization}

We found between-group differences in the FCs of the regions constituting the intrinsic organization.

All group-common TPN component regions showed increased positive or negative (farther from zero) FCs in the depressed group, except for the right MT+, SMA, right dorsal premotor region, and left inferior cerebellum, for which no significant differences were found. Overall, the brain regions that showed significantly increased positive FCs to the seed regions were mainly in the bilateral lateral prefrontal cortices and the IPL. In contrast, the regions that showed significantly increased negative FCs to the seed regions were mainly in the $\mathrm{PCC} / \mathrm{PCu}, \mathrm{MPFC} / \mathrm{mOFC}$, bilateral LPC and anterior temporal regions (Fig. 2A, Table S1 in the supplementary materials).

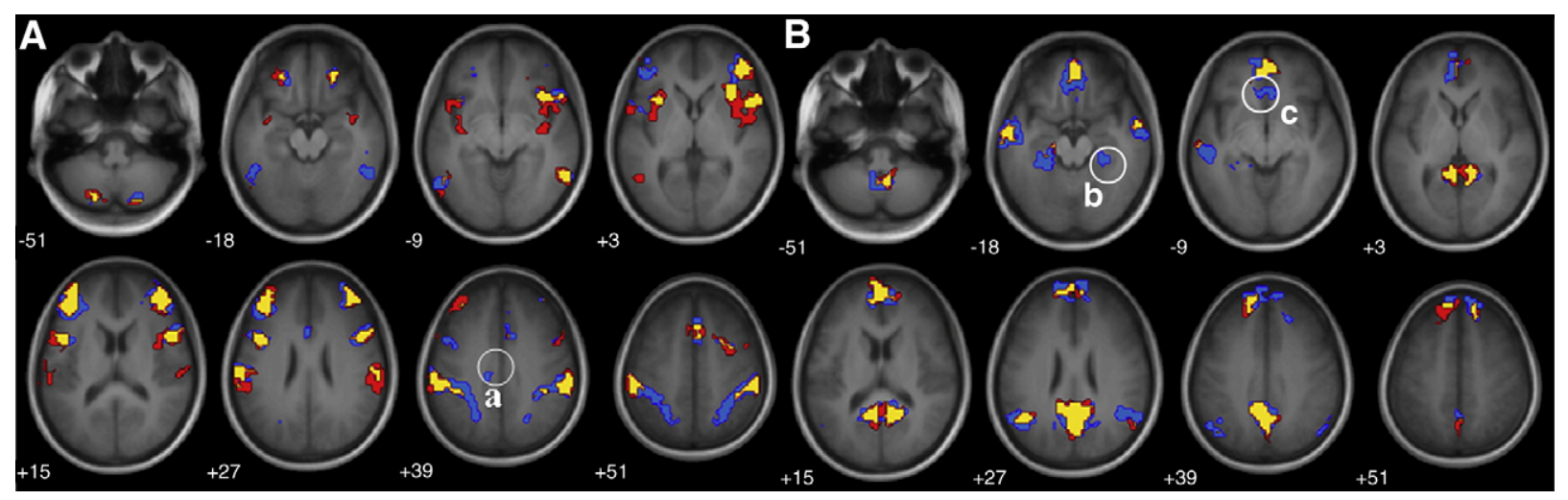

Fig. 1. Regions constituting the task-positive network (A) and task-negative network (B). Red, blue and yellow respectively represent the regions within the control group, within the depressed group and the overlapped regions between the groups. a, b and c respectively represent the left mid-posterior cingulate cortex, the right parahippocampal gyrus and the subgenual cingulated cortex, which only appeared in the depressed group. These regions were superimposed onto a mean anatomical image which was obtained by averaging the normalized high-resolution T1-weighted images across all subjects. (For interpretation of the references to colour in this figure legend, the reader is referred to the web version of this article.) 

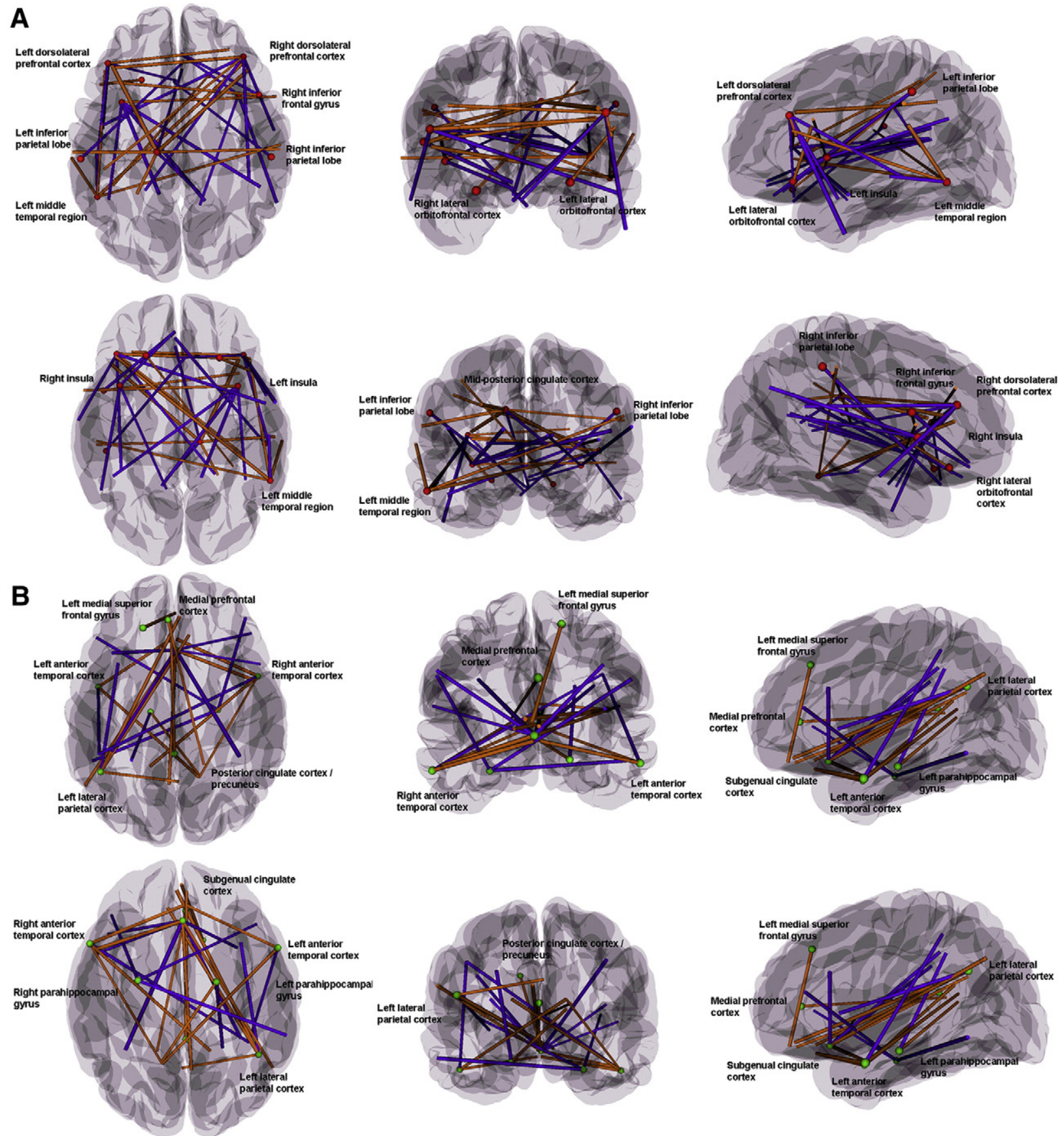

Fig. 2. Altered FCs associated with the regions constituting the task-positive network (A) and in the task-negative network (B) demonstrated in top-, down-, anterior-, posterior-, left- and right-side view. The red balls represent the seed regions constituting the TPN, and the green balls represent the seed regions constituting the TNN. The line represents the altered FC associated with the seed region, with orange for increased positive connectivity and purple for increased negative connectivity in the depressed patients ( $p<0.001$, corrected). No decreased positive or negative connectivity associated with the seed regions were found. For the FCs associated with the cerebellum, please see Tables S1 and S2 in the supplementary materials. (For interpretation of the references to colour in this figure legend, the reader is referred to the web version of this article.)

All group-common TNN component regions showed increased positive or negative FCs in the depressed group, except for the right $\mathrm{mSFG}$, right LPC, and tonsil, in which no significant differences were found. Overall, the brain regions that showed significantly increased positive FCs to the seed regions were mainly in the PCC/PCu and MPFC/mOFC. In contrast the regions that showed significantly increased negative FCs to the seed regions were relatively few and mainly in the left IPL and left lateral prefrontal regions (Fig. 2B, Table S2 in the supplementary materials).

The group-specific network component regions that only appeared in the intrinsic organization of the depressed group all 
showed increased positive or negative FCs in the depressed group. In brief, compared to the healthy subjects, the left midposterior cingulate cortex showed significantly increased positive FCs to the bilateral DLPFC (BA46/10) and IPL, and showed increased negative $\mathrm{FCs}$ to the $\mathrm{PCC} / \mathrm{PCu}$, and $\mathrm{MPFC} /$ mOFC in the depressed patients. The sgACC showed significantly increased positive FCs to the PCC/PCu, left LPC, and right anteriortemporal cortex, and showed increased negative FCs to the bilateral lateral prefrontal cortices and IPL in the depressed patients. The right parahippocampal gyrus showed significantly increased negative FCs to the bilateral lateral prefrontal cortices and left MT+. No other significant differences were observed (Fig. 2, Table S3 in the supplementary materials).

We have schematically summarized our main findings in Fig. 3.

\subsection{Clinical correlates analysis}

Based on the alpha adjusted via the Bonferroni correction for multiple comparisons, we found that the length of the current depressive episode was significantly correlated with the strength of the FCs between the right IFG and the left middle frontal gyrus (partial correlation coefficient $=.76, p<0.05$, corrected) and that the HDRS score was significantly correlated with the strength of the FC between the left DLPFC and the right middle/superior frontal gyrus ( partial correlation coefficient $=$ $-.67, p<0.05$, corrected) after controlling for the age effect. In addition, we observed trends toward significance between the length of the current depressive episode and the strength of the FCs between the right DLPFC and left middle/inferior frontal gyrus (partial correlation coefficient $=.59, p=0.01$, uncorrected) and between the sgACC and the right IFG (partial correlation coefficient $=-.50, p=0.04$, uncorrected).

\section{Discussion}

Several recent studies have addressed the issue of changes in SLFFs in MDD. Anand et al. (2005a) found decreased FCs between the anterior cingulate cortex and the limbic regions in unmedicated depressed patients. These FCs reverted to the normal range after 6 weeks of antidepressant treatment (Anand et al., 2005b). Using an independent component analysis (ICA) approach to extract the default mode network from the low frequency component of BOLD signals, Greicius et al. (2007) showed significantly increased contributions from the subgenual cingulate and thalamus to the network in medicated, but unremitted depressed patients. The present study extended these previous findings by investigating the FCs of the intrinsic organization, i.e. the differences in the component regions in the intrinsic organization and the differences in the FCs associated with these regions between groups. We found that certain regions in the prefrontal, parietal and temporal lobes predominately or only appeared in the intrinsic organization of the depressed group. Furthermore, in the depressed patients all the FCs associated with each component region were found to be increased in either the positive or negative direction. These increased FCs primarily involved regions related to attention and adaptive control within the TPN as well as regions implicated in episodic memory, self-reflective and emotional regulation within the TNN. In addition, increased anti-correlations between the two networks were revealed by the increased negative correlation coefficients between the regions respectively locating in the two networks in MDD (Fig. 3). Furthermore, the strengths of the FCs associated with the lateral prefrontal cortices were found to be correlated with the durations of the depressive episode or the HDRS scores in the depressed patients.

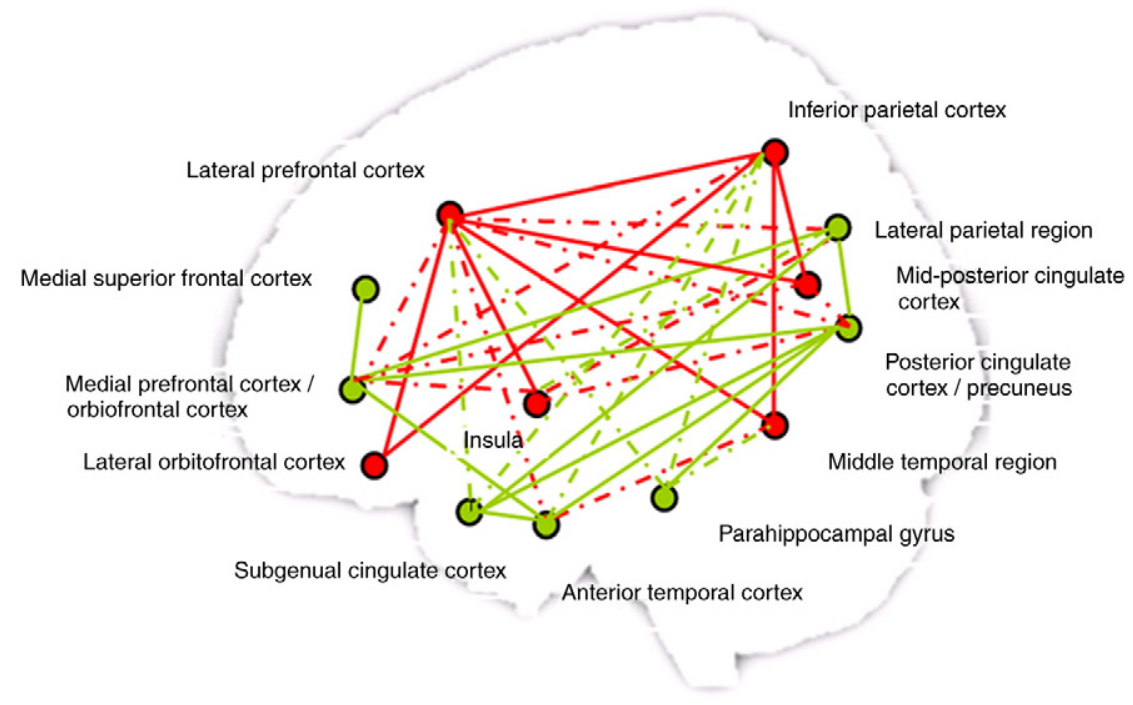

Fig. 3. A schematic figure summarizing the altered FCs within the TPN and TNN and between the two networks in depressed patients. These altered FCs were superimposed onto a semi-transparent three-dimensionally reconstructed cerebral cortex. Red balls represent the seed regions in the TPN and green balls represent the seed regions in the TNN. The red line represents connectivity within the TPN, and the blue line represents connectivity within the TNN. The solid line represents altered positive connectivity, and the dashed line represents altered negative connectivity. The coordinates for each seed region were obtained by computing the centroid of this region. Lines were placed between the coordinates of each seed region and the coordinates of the cluster that shows an altered FC to this seed region. This procedure was implemented by means of visualization toolkit (www.vtk.org) and c ++ . (For interpretation of the references to colour in this figure legend, the reader is referred to the web version of this article.) 
The increased FCs within the TPN in the depressed group were focused in the bilateral lateral prefrontal cortices and IPL, both of which have been implicated in attention and adaptive control (Dosenbach et al., 2007; Fox et al., 2006). In addition, most of the other regions, such as the lateral orbitofrontal cortices, MT+, and insula, which showed increased FCs with these two regions, are also related to attention. The lateral orbitofrontal cortices, in which enhanced resting metabolic activity was found in unmedicated depressed patients (Drevets, 2000), have been suggested as participating in motivation attention (Liddle et al., 2006). MT+ is located in the extended dorsal attention system and is involved in cued attention and working memory (Corbetta and Shulman, 2002; Fox et al., 2006). The insula has been implicated in attention to changes in the "inner milieu" (Craig, 2002). On the other hand, the bilateral lateral prefrontal cortices and IPL and the regions that showed increased FCs with them are implicated in emotion processing. The lateral prefrontal cortex also plays an important role in emotional regulation (Ochsner and Gross, 2005; Ochsner et al., 2004) and participates in attention to emotion and the anticipation of negative emotion (Grimm et al., 2008; Ueda et al., 2003). In major depression, the lateral prefrontal cortex shows increased activation in response to sad autobiographical scripts (Keedwell et al., 2005) and a greater relative recruitment in reappraisal of emotion (Johnstone et al., 2007). In response to sad words, depressed subjects showed more activation in the inferior parietal lobule than did controls (Canli et al., 2004). The lateral orbitofrontal cortices and the anterior insula also participate in emotional responses to stressors (Drevets, 2001). Combining both roles of these regions in attention and emotional processing, it is reasonable to speculate that the focus of attention of depressed patients toward engraved negative emotional experiences may persist even in the absence of current external events. The increased FCs in the regions related to attention may serve as a record or memory of earlier coactivations that resulted from negative experiences or represent a dynamic prediction of expected future coactivations as a result of the overall negative expectations of depressed patients (Fox et al., 2006).

Within the TNN, the increased FCs in the depressed group were focused in the PCC/PCu and MPFC/mOFC. These two regions are core components in the default mode network/ TNN, which has recently been proposed to play a role in constructing dynamic mental simulations based on personal past experiences, such as remembering the past, envisioning the future and generally when imagining alternative perspectives and scenarios to the current situation (Buckner et al., 2008). The two core regions and the regions that showed increased FCs with them in this study, including the LPC, parahippocampus, and anterior temporal cortex, are involved in autobiographical memory retrieval (Svoboda et al., 2006), self-reflective activity and mentalizing (Frith and Frith, 2003), and predicting the future (Addis et al., 2007). Based on previous observations that negative automatic thoughts about one's self, the world, and the future are distinctive and specific to MDD (Clark et al., 1998) and that the frequency of the negative thoughts was increased in MDD (Lau et al., 2007), we speculated that the increased FCs within the TNN may be related to the negative thoughts in MDD. In addition, the $\mathrm{PCC} / \mathrm{PCu}$ and $\mathrm{MPFC} / \mathrm{MOFC}$ also participate in emotional processing. The medial prefrontal regions, especially the sgACC, have been reported to be implicated in emotion processing and in the pathogenesis of mood disorders (Drevets, 2000, 2001). In healthy subjects, the medial prefrontal regions are activated while feeling sadness (Damasio et al., 2000; Harrison et al., 2008; Liotti et al., 2002) and while evaluating the emotional valence of pleasant and unpleasant words (Maddock et al., 2003). In major depression, the medial prefrontal regions show an increased resting metabolism when measured by positron emission tomography (Drevets, 2000); additionally, the increased metabolism declines to the normal range after successful antidepressant treatment (Kennedy et al., 2001; Mayberg et al., 2000). The vPCC provides the code that allows relevant information from visual sensory systems to evaluate emotional content, which is determined from information stored in the sgACC (Vogt et al., 2006). From this point, we can further speculate that the increased FCs within the TNN may reflect an enhanced memory for, and related autonomic activity toward, negative emotional experiences in major depression.

Abnormally increased anti-correlations between the two networks were observed in the depressed group. These increased anti-correlations mainly involved the medial posterior parietal regions and medial prefrontal cortex in the TNN, and the lateral prefrontal cortex and inferior parietal cortex in the TPN. In terms of brain organization, anticorrelations are thought to be as important as correlations (Fox et al., 2005). The correlations within networks may integrate neuronal activities that subserve similar goals or representations, whereas the anti-correlations between networks may differentiate neuronal processes that subserve opposite goals or competing representations (Fox et al., 2005). When the TNN is activated during a period of introspection, the activity of the TPN is attenuated; similarly, when the TPN is activated during a period of extrospection, the activity of the TNN is attenuated. The competition between the networks has been suggested to increase when the cognitive load increases (Rogers et al., 2007). We speculate that the abnormally increased anti-correlations between networks in depressed patients may reflect excessive competition between the mental activities served by the two networks. This may contribute to the concurrence of negative attention bias and negative memory bias in major depression.

The clinical variables were found to be significantly correlated with FCs that are associated with the lateral prefrontal cortices, i.e. a positive correlation with the length of current depressive episode, but a negative correlation with the HDRS score. In addition, we found a trend toward significance between the length of current depressive episode and the FC of the sgACC, which is consistent with a previous study (Greicius et al., 2007). These clinical correlates further suggest that altered resting-state FCs are meaningful in MDD. However, we should be cautious about interpreting the information about the clinical correlates due to the small sample size in this study.

Several additional issues need to be considered in this study. First, in the present study, we used a seed-based FC analysis to characterize functional connectivity. This approach is widely used owing to its inherent simplicity, sensitivity and ease of interpretation, although this approach has its 
disadvantages (for a review, please see Fox and Raichle, 2007). For example, the results obtained by this approach are dependent on an a priori definition of a seed region. This may be one of the reasons that we did not find an increased FCs between the thalamus and the TNN in the depressed group, as had been reported in a previous study (Greicius et al., 2007). In this previous study, ICA was used to characterize functional connectivity. ICA is a model-free approach, which is not limited by a priori predictions, and uses sophisticated algorithms to analyze the entire BOLD data set and decompose it into components that are maximally independent in a statistical sense (Fox and Raichle, 2007). However, determining the number of components and the physiological implication of each component is dependent on a priori criteria (Fox and Raichle, 2007). In the present study, the TNN of healthy subjects detected by FC analysis was similar to what Greicius et al. found by ICA, and our findings that the sgACC only appeared in the network of the depressed group and that the associated FCs were increased are also consistent with their finding (2007). Thus we think that our results are comparable with those of Greicius et al., although there is inconsistency in the thalamus. Besides the methodological issue, the inconsistency may result from patient heterogeneity with respect to the medicated state, the number of episodes and the duration of the current episode, as well as to the severity of the disease. In this study, a group of medication-naïve patients with a first depressive episode and low illness severity were recruited rather than the more severely ill, medicated patients with a longer duration of the current episode in the study by Greicius et al. (2007). In another resting-state study which selected the medial thalamus as seed region, a decreased rather than an increased connectivity associated with the medial thalamus was observed in medication-free depressive patients (Anand et al., 2005a). Further studies are necessary to ascertain the contribution of the thalamus to the resting-state functional organization.

Another issue pertains to the interpretation of SLFF. In the present study, we used a relatively low sampling rate ( $\mathrm{TR}=2 \mathrm{~s}$ ) for multislice acquisitions. Under this sampling rate, cardiac and respiratory fluctuation effects could be aliased into the low frequency ranges at which resting-state connectivity is detected and could reduce the specificity of the connectivity effects (Lowe et al., 1998). However, some studies have confirmed that SLFF is physiologically significant and cannot be attributed to cardiac or respiratory factors (Fox and Raichle, 2007). Furthermore, abnormalities in SLFF correlation that have been observed in different neuropsychiatric illnesses are thought to be a measure of abnormal connectivity that allows researchers to study brain circuitry in pathological conditions as well as treatment effects (Anand et al., 2005a). Although much is known, the pathophysiological basis of altered SLFF correlations needs further clarification.

Finally, in this study, the abnormally increased FCs in the intrinsic organization of MD patients were inferred to be related to the negative bias in major depression based on prior knowledge about the recruited regions; however, due to a lack of related behavioral data in this study, this speculation needs to be validated by future study.

Taking these findings as a whole, we tentatively propose that the medial posterior parietal regions, medial and lateral prefrontal cortices, and inferior parietal cortex may be the "core nodes" of the dysfunctional intrinsic organization of MDD. The increased functional connectivities associated with these core nodes may be the neural basis for the negative bias in emotion, attention and memory observed in major depression. This speculation is partially supported by an even more recent study. In this study, increased activity in the default mode network regions was found during passive and active emotional modulation tasks, suggesting that abnormalities in the default mode network might contribute to deficits in "automatic" and controlled processing of affective stimuli in MDD (Sheline et al., 2009). Graph theory, which has been recently introduced into neuroscience as a means of analyzing brain networks (for a review, see Bullmore and Sporns, 2009), should be used in future studies to identify and classify the core nodes in the intrinsic organization.

In summary, our study suggests the abnormally increased neural sources recruitment of the intrinsic organization, especially in those regions which are known to be related to emotion, attention and memory. These abnormally increased neural activities could provide a neural basis for the negative bias observed in major depression.

\section{Role of funding source}

Funding for this study was provided by the Natural Science Foundation of China, grant nos. 30425004, 30730035, and 30870694, the National Key Basic Research and Development Program (973), grant no. 2007CB512304, and China Postdoctoral Science Foundation (no. 20070420545), which provided support for the data collection.

\section{Conflict of interest}

No conflict declared.

\section{Acknowledgements}

The authors thank Kun Wang for his useful suggestions and Jiefeng Jiang for his help on the artwork. They also express appreciation to Drs. Rhoda E. and Edmund F. Perozzi for the English language assistance.

\section{Appendix A. Supplementary data}

Supplementary data associated with this article can be found, in the online version, at doi:10.1016/j.jad.2009.05.029.

\section{References}

Addis, D.R., Wong, A.T., Schacter, D.L., 2007. Remembering the past and imagining the future: common and distinct neural substrates during event construction and elaboration. Neuropsychologia 45, 1363-1377.

Allen, G., Barnard, H., McColl, R., Hester, A.L., Fields, J.A., Weiner, M.F., Ringe, W.K., Lipton, A.M., Brooker, M., McDonald, E., Rubin, C.D., Cullum, C.M., 2007. Reduced hippocampal functional connectivity in Alzheimer disease. Arch. Neurol. 64, 1482-1487.

Anand, A., Li, Y., Wang, Y., Wu, J., Gao, S., Bukhari, L., Mathews, V.P., Kalnin, A., Lowe, M.J., 2005a. Activity and connectivity of brain mood regulating circuit in depression: a functional magnetic resonance study. Biol. Psychiatry 57, 1079-1088.

Anand, A., Li, Y., Wang, Y., Wu, J., Gao, S., Bukhari, L., Mathews, V.P., Kalnin, A., Lowe, M.J., 2005b. Antidepressant effect on connectivity of the mood-regulating circuit: an FMRI study. Neuropsychopharmacology 30, 1334-1344.

Andreasen, N.C., O'Leary, D.S., Cizadlo, T., Arndt, S., Rezai, K., Watkins, G.L., Ponto, L.L., Hichwa, R.D., 1995. Remembering the past: two facets of episodic memory explored with positron emission tomography. Am. J. Psychiatry 152, 1576-1585. 
Andrews-Hanna, J.R., Snyder, A.Z., Vincent, J.L., Lustig, C., Head, D., Raichle, M.E., Buckner, R.L., 2007. Disruption of large-scale brain systems in advanced aging. Neuron 56, 924-935.

Association, A.P., 2000. Diagnostic and Statistical Manual of Mental Disorders, 4th edition. American Psychiatric Press, Washington, DC.

Biswal, B., Yetkin, F.Z., Haughton, V.M., Hyde, J.S., 1995. Functional connectivity in the motor cortex of resting human brain using echoplanar MRI. Magn. Reson. Med. 34, 537-541.

Bluhm, R.L., Miller, J., Lanius, R.A., Osuch, E.A., Boksman, K., Neufeld, R.W., Theberge, J., Schaefer, B., Williamson, P., 2007. Spontaneous lowfrequency fluctuations in the BOLD signal in schizophrenic patients: anomalies in the default network. Schizophr. Bull. 33, 1004-1012.

Broyd, S.J., Demanuele, C., Debener, S., Helps, S.K., James, C.J., Sonuga-Barke, E.J., 2009. Default-mode brain dysfunction in mental disorders: a systematic review. Neurosci. Biobehav. Rev. 33, 279-296.

Buckner, R.L., Andrews-Hanna, J.R., Schacter, D.L., 2008. The brain's default network: anatomy, function, and relevance to disease. Ann. N. Y. Acad. Sci. 1124, 1-38.

Bullmore, E., Sporns, O., 2009. Complex brain networks: graph theoretical analysis of structural and functional systems. Nat. Rev. Neurosci. 10, 186-198.

Canli, T., Sivers, H., Thomason, M.E., Whitfield-Gabrieli, S., Gabrieli, J.D., Gotlib, I.H., 2004. Brain activation to emotional words in depressed vs healthy subjects. Neuroreport 15, 2585-2588.

Clark, D.A., Beck, A.T., Alford, B.A., 1998. Scientific Foundations of Cognitive Theory and Therapy of Depression. John Wiley, New York.

Corbetta, M., Shulman, G.L., 2002. Control of goal-directed and stimulusdriven attention in the brain. Nat. Rev. Neurosci. 3, 201-215.

Craig, A.D., 2002. How do you feel? Interoception: the sense of the physiological condition of the body. Nat. Rev. Neurosci. 3, 655-666.

Damasio, A.R., Grabowski, T.J., Bechara, A., Damasio, H., Ponto, L.L., Parvizi, J., Hichwa, R.D., 2000. Subcortical and cortical brain activity during the feeling of self-generated emotions. Nat. Neurosci. 3, 1049-1056.

Davidson, R.J., Lewis, D.A., Alloy, L.B., Amaral, D.G., Bush, G., Cohen, J.D., Drevets, W.C., Farah, M.J., Kagan, J., McClelland, J.L., Nolen-Hoeksema, S., Peterson, B.S., 2002. Neural and behavioral substrates of mood and mood regulation. Biol. Psychiatry 52, 478-502.

Dosenbach, N.U., Fair, D.A., Miezin, F.M., Cohen, A.L., Wenger, K.K., Dosenbach, R.A., Fox, M.D., Snyder, A.Z., Vincent, J.L., Raichle, M.E., Schlaggar, B.L., Petersen, S.E., 2007. Distinct brain networks for adaptive and stable task control in humans. Proc. Natl. Acad. Sci. U. S. A. 104, 11,073-11,078.

Drevets, W.C., 2000. Neuroimaging studies of mood disorders. Biol. Psychiatry 48, 813-829.

Drevets, W.C., 2001. Neuroimaging and neuropathological studies of depression: implications for the cognitive-emotional features of mood disorders. Curr. Opin. Neurobiol. 11, 240-249.

Drevets, W.C., Price, J.L., Furey, M.L., 2008. Brain structural and functional abnormalities in mood disorders: implications for neurocircuitry models of depression. Brain Struct. Funct. 213, 93-118.

First, M.B., Spitzer, R.L., Gibbon, M., JBW, W., 1995. Structural Clinical Interview for DSM-IV Axis I Disorder-Patient Edition (SCID-I/P). New York State Psychiatric Institute, New York.

Fox, M.D., Raichle, M.E., 2007. Spontaneous fluctuations in brain activity observed with functional magnetic resonance imaging. Nat. Rev. Neurosci. 8, 700-711.

Fox, M.D., Snyder, A.Z., Vincent, J.L., Corbetta, M., Van Essen, D.C., Raichle, M.E., 2005. The human brain is intrinsically organized into dynamic, anticorrelated functional networks. Proc. Natl. Acad. Sci. U. S. A. 102, 9673-9678.

Fox, M.D., Corbetta, M., Snyder, A.Z., Vincent, J.L., Raichle, M.E., 2006. Spontaneous neuronal activity distinguishes human dorsal and ventral attention systems. Proc. Natl. Acad. Sci. U. S. A. 103, 10,046-10,051.

Fransson, P., 2005. Spontaneous low-frequency BOLD signal fluctuations: an fMRI investigation of the resting-state default mode of brain function hypothesis. Hum. Brain Mapp. 26, 15-29.

Frith, U., Frith, C.D., 2003. Development and neurophysiology of mentalizing. Philos. Trans. R. Soc. Lond., B Biol. Sci. 358, 459-473.

Frith, C.D., Frith, U., 2006. The neural basis of mentalizing. Neuron 50, 531-534.

Genovese, C.R., Lazar, N.A., Nichols, T., 2002. Thresholding of statistical maps in functional neuroimaging using the false discovery rate. Neuroimage $15,870-878$

Greicius, M.D., Krasnow, B., Reiss, A.L., Menon, V., 2003. Functional connectivity in the resting brain: a network analysis of the default mode hypothesis. Proc. Natl. Acad. Sci. U. S. A. 100, 253-258.

Greicius, M.D., Flores, B.H., Menon, V., Glover, G.H., Solvason, H.B., Kenna, H., Reiss, A.L., Schatzberg, A.F., 2007. Resting-state functional connectivity in major depression: abnormally increased contributions from subgenual cingulate cortex and thalamus. Biol. Psychiatry 62, 429-437.

Grimm, S., Beck, J., Schuepbach, D., Hell, D., Boesiger, P., Bermpohl, F., Niehaus, L., Boeker, H., Northoff, G., 2008. Imbalance between left and right dorsolateral prefrontal cortex in major depression is linked to negative emotional judgment: an fMRI study in severe major depressive disorder. Biol. Psychiatry 63, 369-376.

Gusnard, D.A., Raichle, M.E., 2001. Searching for a baseline: functional imaging and the resting human brain. Nat. Rev. Neurosci. 2, 685-694.

Gusnard, D.A., Akbudak, E., Shulman, G.L., Raichle, M.E., 2001. Medial prefrontal cortex and self-referential mental activity: relation to a default mode of brain function. Proc. Natl. Acad. Sci. U. S. A. 98, 4259-4264.

Hamilton, M., 1960. A rating scale for depression. J. Neurol. Neurosurg. Psychiatry 23, 56-62.

Harrison, B.J., Pujol, J., Ortiz, H., Fornito, A., Pantelis, C., Yucel, M., 2008. Modulation of brain resting-state networks by sad mood induction. PLoS ONE 3, e1794.

Jiang, A., Kennedy, D.N., Baker, J.R., Weisskoff, R.M., Tootell, R.B.H., Woods, R.P. Benson, R.R., Kwong, K.K., Brady, T.J., Rosen, B.R., 1995. Motion detection and correction in functional MR imaging. Hum. Brain Mapp. 3, 224-235.

Johnstone, T., van Reekum, C.M., Urry, H.L., Kalin, N.H., Davidson, R.J., 2007. Failure to regulate: counterproductive recruitment of top-down prefrontal-subcortical circuitry in major depression. J. Neurosci. 27, 8877-8884.

Keedwell, P.A., Andrew, C., Williams, S.C., Brammer, M.J., Phillips, M.L., 2005. A double dissociation of ventromedial prefrontal cortical responses to sad and happy stimuli in depressed and healthy individuals. Biol. Psychiatry 58, 495-503.

Kennedy, D.P., Courchesne, E., 2008. The intrinsic functional organization of the brain is altered in autism. Neuroimage 39, 1877-1885.

Kennedy, S.H., Evans, K.R., Kruger, S., Mayberg, H.S., Meyer, J.H., McCann, S., Arifuzzman, A.I., Houle, S., Vaccarino, F.J., 2001. Changes in regional brain glucose metabolism measured with positron emission tomography after paroxetine treatment of major depression. Am. J. Psychiatry 158, 899-905.

Lau, M.A., Christensen, B.K., Hawley, L.L., Gemar, M.S., Segal, Z.V., 2007. Inhibitory deficits for negative information in persons with major depressive disorder. Psychol. Med. 37, 1249-1259.

Leppanen, J.M., 2006. Emotional information processing in mood disorders: a review of behavioral and neuroimaging findings. Curr. Opin. Psychiatry 19, 34-39.

Liddle, P.F., Laurens, K.R., Kiehl, K.A., Ngan, E.T., 2006. Abnormal function of the brain system supporting motivated attention in medicated patients with schizophrenia: an fMRI study. Psychol. Med. 36, 1097-1108.

Liotti, M., Mayberg, H.S., McGinnis, S., Brannan, S.L., Jerabek, P., 2002. Unmasking disease-specific cerebral blood flow abnormalities: mood challenge in patients with remitted unipolar depression. Am. J. Psychiatry $159,1830-1840$

Lowe, M.J., Mock, B.J., Sorenson, J.A., 1998. Functional connectivity in single and multislice echoplanar imaging using resting-state fluctuations. Neuroimage 7, 119-132.

Maddock, R.J., Garrett, A.S., Buonocore, M.H., 2003. Posterior cingulate cortex activation by emotional words: fMRI evidence from a valence decision task. Hum. Brain Mapp. 18, 30-41.

Maldjian, J.A., Laurienti, P.J., Kraft, R.A., Burdette, J.H., 2003. An automated method for neuroanatomic and cytoarchitectonic atlas-based interrogation of fMRI data sets. Neuroimage 19, 1233-1239.

Mayberg, H.S., 2003. Modulating dysfunctional limbic-cortical circuits in depression: towards development of brain-based algorithms for diagnosis and optimised treatment. Br. Med. Bull. 65, 193-207.

Mayberg, H.S., Brannan, S.K., Tekell, J.L., Silva, J.A., Mahurin, R.K., McGinnis, S., Jerabek, P.A., 2000. Regional metabolic effects of fluoxetine in major depression: serial changes and relationship to clinical response. Biol. Psychiatry 48, 830-843.

Ochsner, K.N., Gross, J.J., 2005. The cognitive control of emotion. Trends Cogn. Sci. 9, 242-249.

Ochsner, K.N., Ray, R.D., Cooper, J.C., Robertson, E.R., Chopra, S., Gabrieli, J.D., Gross, J.J., 2004. For better or for worse: neural systems supporting the cognitive down- and up-regulation of negative emotion. Neuroimage 23, 483-499.

Phillips, M.L., Drevets, W.C., Rauch, S.L., Lane, R., 2003. Neurobiology of emotion perception II: implications for major psychiatric disorders. Biol. Psychiatry 54, 515-528.

Press, W.H., Teukolsky, S.A., Vetterling, W.T., Flannery, B.P. (Eds.), 1992 Numerical Recipes in C, Second Ed. Cambridge University Press, UK.

Raichle, M.E., MacLeod, A.M., Snyder, A.Z., Powers, W.J., Gusnard, D.A., Shulman, G.L., 2001. A default mode of brain function. Proc. Natl. Acad. Sci. U. S. A. 98, 676-682.

Rogers, B.P., Morgan, V.L., Newton, A.T., Gore, J.C., 2007. Assessing functional connectivity in the human brain by fMRI. Magn. Reson. Imaging 25, 1347-1357.

Seminowicz, D.A., Mayberg, H.S., McIntosh, A.R., Goldapple, K., Kennedy, S. Segal, Z., Rafi-Tari, S., 2004. Limbic-frontal circuitry in major depression: a path modeling metanalysis. Neuroimage 22, 409-418. 
Sheline, Y.I., Barch, D.M., Price, J.L., Rundle, M.M., Vaishnavi, S.N., Snyder, A.Z Mintun, M.A., Wang, S., Coalson, R.S., Raichle, M.E., 2009. The default mode network and self-referential processes in depression. Proc. Natl. Acad. Sci. U. S. A. 106, 1942-1947.

Shulman, G.L., Fiez, J.A., Corbetta, M., Buckner, R.L., Miezin, F.M., Raichle, M.E. Petersen, S.E., 1997. Common blood flow changes across visual tasks: II. Decreases in cerebral cortex. J. Cogn. Neurosci. 9, 648-663.

Sonuga-Barke, E.J., Castellanos, F.X., 2007. Spontaneous attentional fluctuations in impaired states and pathological conditions: a neurobiological hypothesis. Neurosci. Biobehav. Rev. 31, 977-986.

Svoboda, E., McKinnon, M.C., Levine, B., 2006. The functional neuroanatomy of autobiographical memory: a meta-analysis. Neuropsychologia 44, 2189-2208.

Uddin, L.Q., Clare Kelly, A.M., Biswal, B.B., Xavier Castellanos, F., Milham, M.P., 2009. Functional connectivity of default mode network components: correlation, anticorrelation, and causality. Hum. Brain Mapp. 30, 625-637.
Ueda, K., Okamoto, Y., Okada, G., Yamashita, H., Hori, T., Yamawaki, S., 2003. Brain activity during expectancy of emotional stimuli: an fMRI study. Neuroreport 14, 51-55.

Vogt, B.A., Vogt, L., Laureys, S., 2006. Cytology and functionally correlated circuits of human posterior cingulate areas. Neuroimage 29, 452-466.

Wang, L., Zang, Y., He, Y., Liang, M., Zhang, X., Tian, L., Wu, T., Jiang, T., Li, K., 2006. Changes in hippocampal connectivity in the early stages of Alzheimer's disease: evidence from resting state fMRI. Neuroimage 31, 496-504.

Zhou, Y., Liang, M., Jiang, T., Tian, L., Liu, Y., Liu, Z., Liu, H., Kuang, F., 2007a. Functional dysconnectivity of the dorsolateral prefrontal cortex in firstepisode schizophrenia using resting-state fMRI. Neurosci. Lett. 417, 297-302.

Zhou, Y., Liang, M., Tian, L., Wang, K., Hao, Y., Liu, H., Liu, Z., Jiang, T., 2007b. Functional disintegration in paranoid schizophrenia using resting-state fMRI. Schizophr. Res. 97, 194-205. 